\title{
Time for action- oxytocin \& uterotonics are life-saving AND dangerous: a commentary
}

\author{
Deborah Armbruster ${ }^{1}$ \\ ${ }^{1}$ United States Agency for International Development
}

August 15, 2021

\section{Time for action- oxytocin \& uterotonics are life-saving AND dangerous: a commentary}

Deborah Armbruster

United States Agency for International Development

Washington, DC (currently based in Indonesia)

Global Health Bureau/Maternal, Child and Nutrition Office/Maternal and Newborn Division

$+1202746-5453$

darmbruster@usaid.gov

\section{Time for action- oxytocin \& uterotonics are lifesaving AND dangerous: a commentary}

Marx-Delaney, et al have written an excellent article documenting high prevalence of oxytocin misuse and associated adverse outcomes during the intrapartum period in India. They explain how use of the Safe Childbirth Checklist and coaching led to decreased misuse of oxytocin for augmentation and increased use of oxytocin postpartum to prevent postpartum hemorrhage ( $\mathrm{PPH})$. Both effects persisted during the six month follow-up period during which coaching ceased. Although encouraging, changes in behavior of women, providers and health systems will be difficult given the long-term cultural acceptance of oxytocin use during labour. In northern India, research between 1983-1989 by Jeffrey (Jeffrey, et al, Labour Pains and Labour Power. London:Zed Books, 1989, 111-112) found that 15\% of labouring women received oxytocin from government pharmacists. Similarly, in Van Hollen's 2003 study (Van Hollen C, Medical Anthropology Quarterly, 2003, 17(1), 49-77), providers stated that women did not just ask but demanded oxytocin in southern India. A 2011 study in four districts in India documented augmentation rates from 53-93\%, many of which were administered via intramuscular injection or intravenous push (Stanton, et al, IJGO, 2014, 127(1), 25-30). Augmentation rates ranging from 38\% in Benin (2006) to 32\% in both Honduras and Nicaragua (2006) (Lovold, et al, IJGO, 2008, 103, 276-282) imply that high rates and misuse of oxytocin for labour augmentation, with its documented adverse outcomes, is a global issue.

Given the widespread cultural acceptance of oxytocin use by providers and patients alike, two significant issues are raised. First, the well-researched and appropriate recommendations proposed in numerous studies have been largely ignored. Second, the introduction of a new uterotonic and the increased availability of another adds to the complexity of the augmentation landscape, potentially exacerbating the problem, and risk to women and fetuses.

WHO produced recommendations for labour augmentation in 2014. However, other recommendations have not been implemented, such as ensuring awareness of the current prevalence of augmentation and intrapartum uterotonic use in general, and documenting maternal and perinatal outcomes associated with these practices. 
Newer uterotonics, such as misoprostol and heat stable carbetocin (HSC), are potentially lifesaving drugs to address PPH. Neither are appropriate for augmentation, and confusion about their intrapartum safety profile could put women and fetuses at risk. Inadequate messaging surrounding the recent introduction of HSC has left many believing HSC to be a 1:1 substitute for oxytocin. In reality, while an excellent choice for PPHprevention, its longer half life (30-40 minutes versus 1-6 minutes for oxytocin) makes it dangerous for augmentation.

A systematic review of induction and augmentation is currently underway to help us understand the prevalence of these practices and the extent of adverse outcomes. It is time that the global community creates clear, consistent, evidence-based messaging and clinical protocols to begin to address this problem. Countries also need to contextualize and supplement existing research and guidelines to ensure their unique needs are being met. Urgent action is needed to decrease the misuse of uterotonics in labour for the health and well-being of the unborn and their mothers.

This commentary reflects the views of the author and does not necessarily reflect the views of the U.S. Agency for International Development or the U.S. government.

Acknowledgements: Sincere thanks to Emma Clark, Cindy Stanton and Mary Ellen Stanton for their reviews and edits to the Commentary 